\author{
Visualization of Scientific Data \\ Work Performed for X-3 \\ by \\ Applied Computing Systems, Inc.
}

Final Report

September 29, 1989

Applied Computing Systems, Inc., started work on the computer codes for visualization of X-3 scientific data on the Silicon Graphics 4D70/GT workstation on May 18, 1989. There were two separate codes, a 2-D and a 3-D version, that had been developed by X-3 over approximately the previous six months. Work was performed under three successive tasking orders, and the specific accomplishments are summarized below as excerpts from the monthly reports that had been submitted while the work was being performed.

Installing the video equipment was listed as a subtask in each of the three task orders. However, the video equipment did not arrive until the start of task 3 , so that work was delayed until the very end.

\title{
TASK 1
}

During May:

(1) We created a Revision Control System (RCS) directory and placed the codes under the control of RCS. RCS is a public domain source code control system that is distributed with the Silicon Graphics software, and is available on most UNIX computer systems. It allows for easy maintenance of multiple versions of codes along with textual information describing each version. To save space it only saves the differences between versions, yet any version saved may be quickly retrieved.

(2) The code had been developed very quickly with the ideas of achieving maximum functionality in the shortest time, and of demonstrating different concepts using rapid prototyping. Because of this, the code was in need of some cleanup. We commented all variables, modularized the code, and did some restructuring and cleanup.

(3) ACS is using a Silicon Graphics 4D20 personal IRIS for code development. Since this is a slower machine than the 4D70GT at X-Division, and since we only have 8 megabytes of memory versus the 16 megabytes at X-Division, we did several things to speed up the display of data:

(a) We added the capability to read in a subset of the data that fits in memory. This was necessary since the program and data together are larger than our available memory, so paging was excessive and program startup and operation were very sluggish.

(b) We added the capability to compile data into code (data statements). Code that is produced in this way generates an executable that starts up much more quickly. This is useful for program development, since it is much quicker to test new ideas. It is also useful for giving demonstrations of the code since the program starts up quickly.

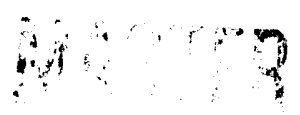


During the month of June, the following items were accomplished:

(1) The event loop was modified so that mouse clicks for menu selections happen quicker, and the event loop blocks so that the program lies dormant when no new action has been requested by the user.

(2) A 4sight interface was set up based on the examples in 4Dgifts to provide a more functional, attractive, and modifiable window interface. This interface also has the necessary hooks for performing the propagation of the shell variables from the authorization function.

(3) A color editor was started on that makes it easier to select desired colors for color maps or other purposes. It shows in color the range of colors possible if red, green, or blue is preselected. It also will allow for the selection of the predefined colors by name from the GL, NeWS, and X11. A gray scale will also be provided.

(4) The twodimm program was modified to use either mapped or 24-bit color, and a lighting model was added. A routine for the numerically robust calculation of surface normals was used for this purpose.

(5) Pull-right menus were implemented in the GL for time selection of frames. This could be done with a different method such as a scrolling window, since the range of times is potentially unlimited.

Work was started on a method to allow constructing a composite time selection menu that allows the user to select parts of several different data dump files for display in a single animation sequence.

(6) A "soft" dialbox that is compatible with the dialbox was constructed for man-machine interface purposes.

(7) A program was constructed that uses both the GL and NeWS together. This program showed that it should be possible to use NeWS for the man-machine interface and the GL for the 3-D graphics. The issues of using NeWS and/or the GL were summarized and written down. Based on this, it was decided to use NeWS, where appropriate, for man-machine interface functions.

Interprocess communication tests were constructed using both shared memory and sockets. These will be used in later work.

(8) A crude thinning algorithm was started on, so that adjustments could be made with quick visual feedback on a realistic image.

(9) Backups of the IRIS system were performed, and the procedure for doing this was documented.

A user interface was started on that can provide a framework for satisfying current and future interface requirements for the visualization work. The work was done in 4Sight (NeWS) for flexibility, operability in a network environment, and portability. Several new menu subclasses were defined and implemented to achicve the user interface that was specified by Phil Romero. Since the work was done in NeWS, which provides an object-oriented programming environment, this was able to be accomplished by "inheriting" most of the properties of Silicon Graphics 4Sight menus and changing only those features that were required to be changed. 


\section{TASK 2}

During the month of July, the following specific items were accomplished:

(1) Four new 4Sight menu classes were defined and implemented: Litemenu2, SGIMenu2, HighlightMenu, and StayupHighlightMenu.

The first two classes were added to allow the menus to display in the color planes instead of the overlay plane. This allows the full use of colors - specifically this was required to be able to show color highlighting, since the overlay plane only allows for a limited amount of color.

The last two classes were added to allow for stayup, as opposed to popup, menus, and to allow for selection of multiple items with color highlighting of those items that are selected. Also added was the capability to select multiple items by dragging the mouse with the left button depressed.

(2) An interface for running the three MESA visualization codes was constructed with a master button which, when selected, leads the user through the steps to view his data files. This code is in the source code files "mesafiles" and "mesamenu."

(3) An interface for selection of times and objects from the data files was constructed. The source code files are "Ismenu" and "mkindex." A selection of objects and times generates an index into the data files that will be able to be read by the MESA visualization codes.

(4) Work was started on a lighting model and perspective preview interface using separate windows for these two functions. Since it is desirable to potentially have several MESA visualization codes running concurrently, the interface will be a separate UNIX process that communicates with those codes using network sockets.

(5) Grey scale and NeW/S/X11 color palettes were added to the color editor.

The menu system interface to the three $X-3$ visualization codes was continued, and the menu system was tied into the three dimensional code. This allows the users to easily select sets of times and objects from different data files, and then view the selected objects with the visualization code. Also, a prototype program was constructed to allow interactive modification of lighting, material and perspective parameters with visual feedback, while simultaneously viewing the rendered scene of interest.

During the month of August, the following specific items were accomplished:

(1) The menu interface to the three MESA visualization codes was expanded. The abilities to save or run the current setup and to undo choices were added to the menus, and cosmetic changes were added as well. The menus were made to work appropriately for the two dimensional and voxel versions of the programs, since there is only one "object" involved in those programs. Text input was added to the interface using 4 Sight text items, which caused a few technical problems that had to be solved.

The new color editor was added to the two dimensional code.

(2) Software was written to allow for establishing a communication channel between a 4 Sight process and a process using the SGI Graphics library. This will be used for enhancing the capabilities of menus in the 3D code, (what Phil Romero is calling the menu tool kit), and for the optical disk and VCR control panel code. 
(3) A lighting model and perspective previewer was prototyped, using code that Phil brought back from his SGI class. The prototype allows the user to interactively see and modify the lighting and perspective parameters while also viewing his rendered scene. We will interface this to the three dimensional code, and if there is interest it can be enhanced further.

\section{TASK 3}

During the month of September, we developed a "soft" control panel for the optical disk recorder and implemented a menu toolkit for use with menus created with the Silicon Graphics graphics library. We also discovered the link between graphics library windows and 4 Sight windows. This makes it possible to perform 4Sight (PostScript) graphics, with some restrictions, in conjunction with graphics library three dimensional graphics.

Specifically, the following items were accomplished:

(1) An optical disk recorder control panel was implemented in 4 Sight for controlling the optical disk recorder from the screen of the Silicon Graphics workstation. The panel contains two logically different sections. The first section duplicates the important controls of the recorder and the remote control. The second section implements higher level functionality such as playing an animation of a range of frames, by sending the recorder the individual steps necessary to enable the recorder to do this. It is easy to add new programs, as needed. Help messages were added to make the interface relatively self contained for the users.

(2) Menu additions were made to the visualization interface to support some new features that were requested. These include the capability to obtain files from mass storage, and to modify the "times" menu to be able to display more time entries than will fit vertically on the screen.

(3) A menu toolkit was implemented to extend the functionality of the Silicon Graphics graphics library. While certain specific functions were implemented, the methodology used makes it easy for someone familiar with NeWS or 4 Sight to change or extend virtually any aspect of the graphics library menu capabilities.

The graphics library uses 4 Sight to implement menus and windows. However, since the $C$ and CPS source code for that library is not available to us, the difficult part was to discover how this was done without having all of the source code (the 4Sight part of the code is available in /usr/NeWS/lib/NeWS).

(4) It was discovered how the graphics library uses 4 Sight to implement windows. Again, the problem was that the $C$ and CPS source code is not available to us. The graphics library sets a boolean PostScript variable "GLWindow". If this variable is set, then the standard 4Sight PostScript graphics to the window Joes not work correctly. We attempted to subvert the system by unsetting the variable, doing the graphics, and then resetting the variable. This enabled the graphics to work, but then the 4 Sight server crashed after a short time. So this approach does not look promising.

However, since we have discovered the necessary links between the graphics library and 4 Sight, it is possible to do PostScript graphics on a graphics library window by making each object of the graphics be a separate canvas shaped like the object. This means that it is possible to do powerful PostScript annotation on a graphics library window, although not as easily as one would have hoped. 
(5) The color editor that was programmed earlier in the project was tied into the "mvimm" three dimensional material interface program.

(6) All source code was turned over to Phil Romero and should be available on the Silicon Graphics workstation in X3.

\section{DISCLAIMER}

This report was prepared as an account of work sponsored by an agency of the United States Government. Neither the United States Government nor any agency thereof, nor any of their employees, makes any warranty, express or implied, or assumes any legal liability or respunsibility for the accuracy, completeness, or usefulness of any information, apparatus, product, or process disclosed, or represents that its use would not infringe privately owned rights. Reference herein to any specific commercial product, process, or service by trade name, trademark, manufacturer, or otherwise does not necessarily constitute or imply its endorsement, recommendation, or favoring by the United States Government or any agency thereof. The views and opinions of authors expressed herein do not necessarily state or reflect those of the United States Government or any agency thereof. 


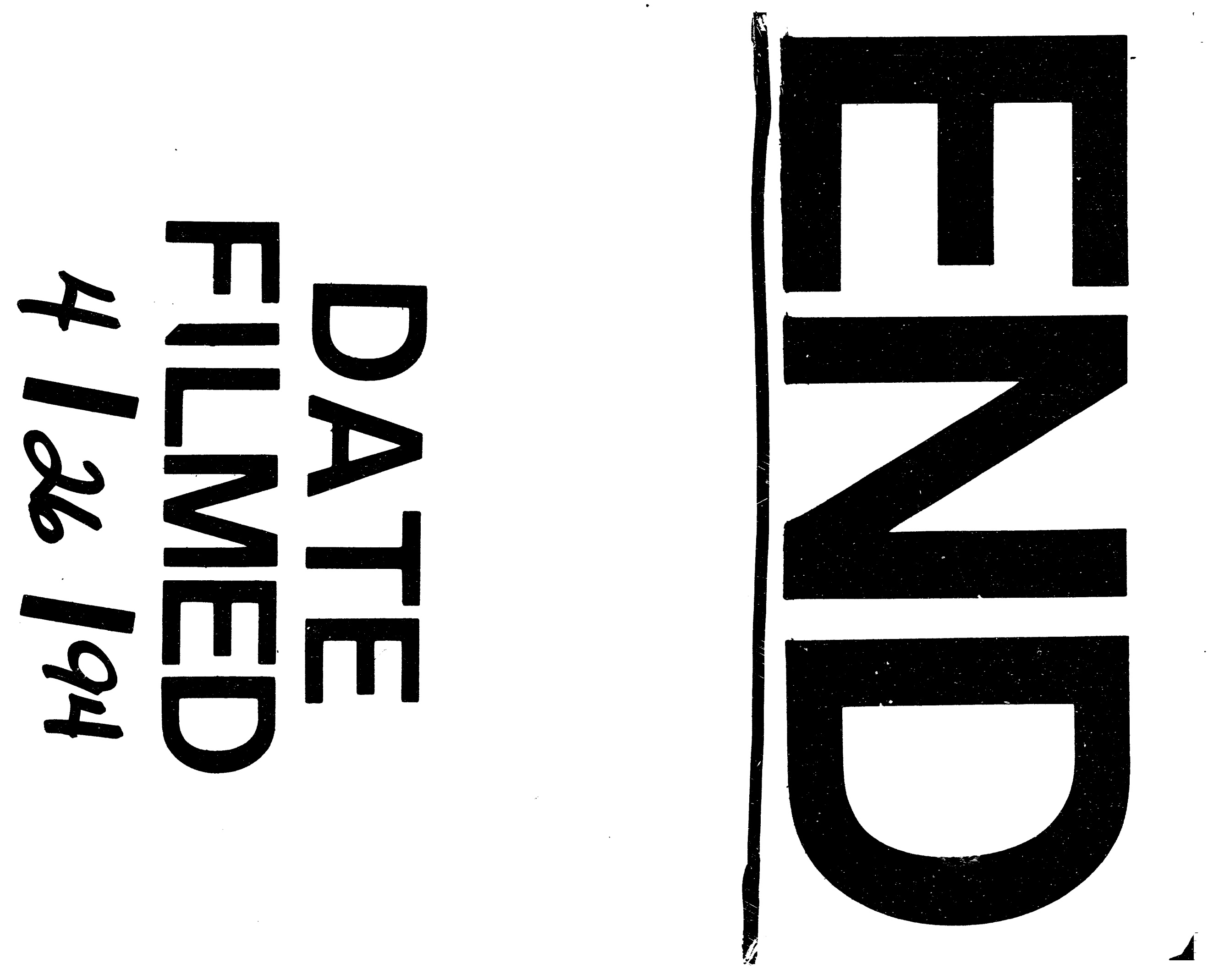


